\title{
Nuevo modelo de aerogenerador
}

\section{Boris Voronin \\ Víctor Rosario Nuño-Sánchez}

\author{
Universidad de Guadalajara \\ Guadalajara, Av. Juárez núm. 976, \\ Colonia Centro, C.P. 44100, Jalisco, México. \\ MÉXICO
}

Tel. +52 (33) 31342222

correos electrónicos (emails):

boris.voronine@cucei.udg.mx

victor.nuno.sanchez@gmail.com

Recibido 08-05-2019, aceptado 23-08-2019.

\section{Resumen}

El artículo presenta un resumen de muchos años de pruebas realizadas por los autores en busca del proyecto más eficiente y, al mismo tiempo, más económico de un aerogenerador. Este artículo expone los resultados de las pruebas de varios modelos a escala en un túnel de viento.

Se detalla un esquema general de un aerogenerador equipado con dos turbinas de aire ubicadas en los extremos de la góndola. Se describe el diseño de la turbina del aerogenerador y sus palas, siendo estas sus elementos principales. Además, se muestran los resultados de una prueba comparativa del modelo de aerogenerador equipado con una y con dos turbinas montadas en un mismo eje y se observa la dependencia de la eficiencia del generador de los parámetros de las palas. Se presenta un nuevo esquema de multiplicador de velocidad del eje del generador eléctrico y un método para generar energía eléctrica en un amplio rango de velocidad del viento.

Este material está destinado a ser utilizado por ingenieros que trabajan en la industria energética dedicada al diseño y fabricación de generadores eólicos de media y alta potencia.

Palabras clave: energía eólica, turbina, álabe, multiplicador de velocidad.

\section{Abstract (A New Model of Wind Turbine)}

The paper presents a summary of many years of tests carried out by the authors searching for the most efficient and, at the same time, the most economical project of a wind generator.
This paper presents the results of the tests of several scale models in a wind tunnel.

The paper shows a general scheme of a wind generator equipped with two air turbines located at the ends of the nacelle. The design of the wind turbine and its blades as main elements are described. The results of a comparative test of the generator model equipped with one and two turbines mounted on the same shaft and the dependence of the generator efficiency on the parameters of the blades are also presented. Anew multiplier scheme for the speed of the electric generator shaft and a method to control the energy of the wind generator in a wide range of speed is included.

The material is intended to be used by engineers working in the energy industry dedicated to the design and manufacture of medium and high power wind turbines.

Index terms: wind energy, turbine, blade, speed multiplier.

\section{Introducción}

Expertos alrededor de todo el mundo señalan que el calentamiento global experimentado por el planeta Tierra, está estrechamente relacionado con la sobreexplotación de los recursos naturales, lo que a su vez lleva al agotamiento de las fuentes de energía. Por lo tanto, sin el desarrollo de fuentes de energía alternativas, es casi imposible predecir el futuro de la humanidad. Una de las fuentes de energía más prometedoras es la energía del viento, la cual no tiene forma acumulativa, por lo tanto, es inagotable.

No hay duda de que las turbinas, que convierten la energía cinética del viento en mecánica, son la parte principal de los aerogeneradores. Es por eso que los autores de este artículo se han dedicado a la búsqueda de su configuración más eficiente.

\section{Desarrollo del problema}

\subsection{Diseño general de aerogenerador}

En estos estudios, se dio prioridad a los aerogeneradores equipados con dos turbinas montadas en los extremos de la góndola, una a barlovento y otra a sotavento, que presumiblemente pueden aumentar la potencia del generador $\mathrm{y}$, por consiguiente, la energía eléctrica generada [1], [2], [3], [4]. Esta suposición se sustenta en la ley de Betz. Esta ley establece que una sola turbina eólica puede convertir el 59.3\% de la energía cinética del viento en energía mecánica. Por lo tanto, si, según la ley 
de Betz, la turbina de barlovento puede usar 59.3\% de la energía cinética del viento, entonces la turbina de sotavento también puede usar $59.3 \%$ de la energía cinética del viento, no utilizada por la turbina de barlovento. Por lo tanto, un aerogenerador equipado con dos turbinas, una de barlovento y otra de sotavento, teóricamente puede convertir $(0.593+(1-0.593) 0.593) 100=83.435 \%$ de la energía cinética de viento en la energía mecánica.

Es decir, el uso de la energía cinética del viento puede incrementarse en un $[(0.83435-0.593) / 0.593] 100=40.7 \%$ [2] Según las pruebas realizadas en un túnel de viento, el incremento de la potencia de un aerogenerador equipado con dos turbinas, con respecto al aerogenerador de una sola turbina de barlovento fue del $38 \%$ al $43 \%$, lo que puede considerarse como una completa coincidencia con los cálculos presentados.

Aquí se propone un modelo de aerogenerador que, según la opinión de los autores, es más sencillo y posiblemente más económico que los que se utilizan actualmente en la industria energética. Además, el aerogenerador propuesto puede generar energía eléctrica en un rango muy amplio de velocidades del viento, lo cual no está dentro de la capacidad de los aerogeneradores modernos. En la Fig. 1, Fig. 2 y Fig. 3 se muestran vistas principales del aerogenerador.

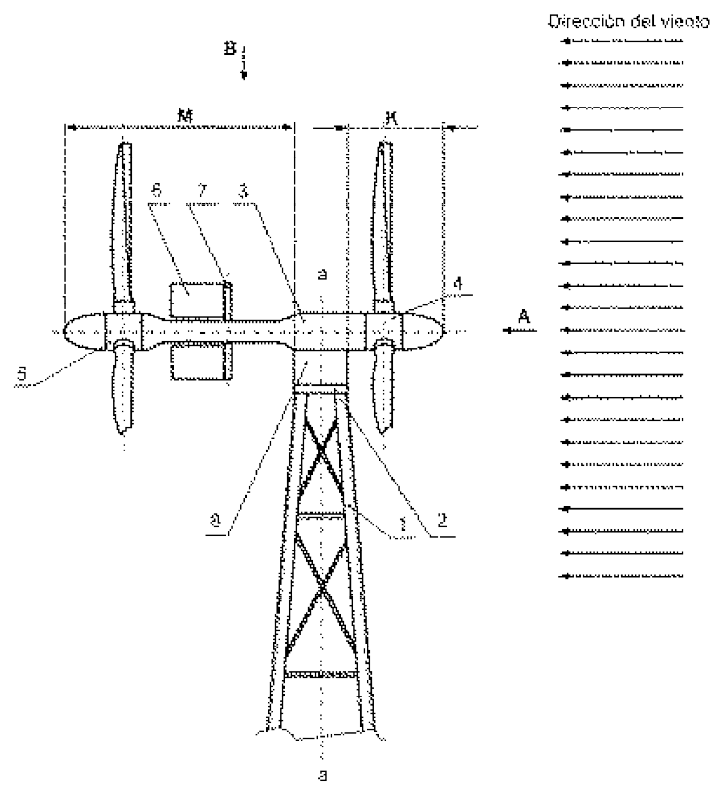

Fig. 1. Vista lateral que es la vista principal del aerogenerador que se presenta.

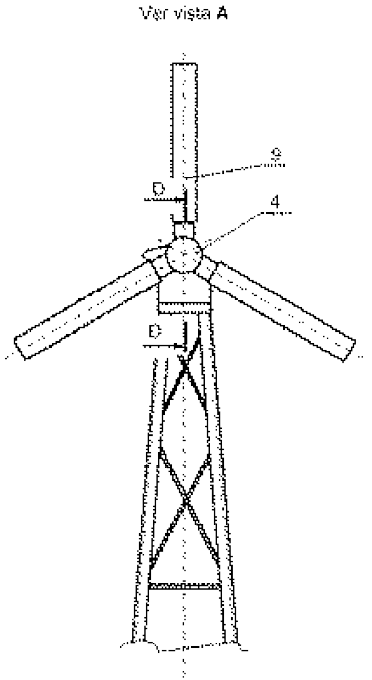

Fig. 2. Vista frontal del aerogenerador.

El aerogenerador está compuesto de la torre 1, encima de la cual se instala la base 2. En la base 2, se instala la góndola 3 con la capacidad de girar libremente alrededor de un eje que coincide con el eje $a$ - $a$ de la torre y de modo que el centro de gravedad esté en el eje de rotación de la góndola. En los extremos de la góndola 3 se instalan dos turbinas: una de barlovento 4 y otra de sotavento 5 . Las turbinas 4 y 5 se instalan de manera que las palas de la turbina de sotavento 5 y de la de barlovento 4 tengan la misma proyección sobre el plano perpendicular al eje de rotación, con lo que desde la vista frontal se observa como si fuera una sola turbina. Las turbinas 4 y 5 tienen el mismo diseño, el mismo sentido de rotación y la misma velocidad angular. Las turbinas de barlovento 4 y de sotavento 5 se instalan en los extremos de las flechas, las cuales se colocan dentro de la góndola 3 y mediante el multiplicador de velocidad 8 [6] transmiten un movi-

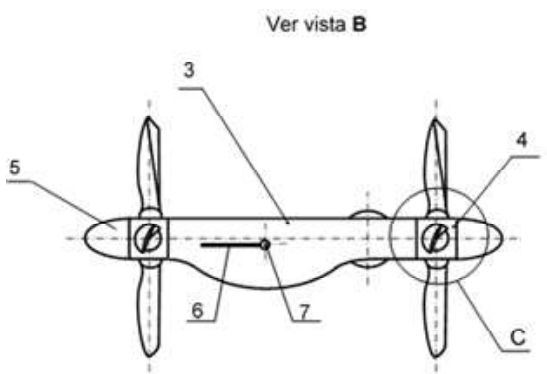

Fig. 3. Vista superior. 
miento giratorio a la flecha del generador eléctrico. El aerogenerador está equipado con un sistema para cambiar automáticamente la orientación de la góndola con respecto a la dirección del viento [1].

\subsection{Diseño de la góndola}

La góndola 3 condicionalmente se divide en dos partes con respecto al eje de la torre $a$ - $a$ : la parte de barlovento $\mathrm{K}$, al final de la cual está instalada la turbina de barlovento 4 , y la parte de sotavento $\mathrm{M}$, al final de la cual está instalada la turbina de sotavento 5 (véase Fig. 1). La parte de sotavento se realiza más larga que la de barlovento, pero respetando la condición que la resistencia lateral al flujo de aire de ambas partes debe ser igual, la parte de sotavento de la góndola 3 es hecha en forma aerodinámica (véase la Fig. 1 y Fig. 3). La longitud de la góndola determina la distancia entre las turbinas, por lo que esto fue uno de los puntos de observación en los estudios. El estudio de la influencia de la distancia entre las turbinas, mostró que una distancia corta entre ellas (aproximadamente $1 / 2$ del diámetro de una turbina), o una distancia de 2 a 4 veces mayor, reflejan una diferencia en la potencia que prácticamente no es perceptible.

Para que el análisis experimental fuera más flexible y tuviera mayor variedad, los cubos de las turbinas se hicieron de modo para que se pudiera instalar dos o cuatro palas. Esto hizo posible verificar el efecto del desplazamiento angular de una turbina con respecto a otra alrededor de su eje geométrico común sobre la potencia del aerogenerador. Por ejemplo, para un generador con 2 turbinas de cuatro palas, si el desplazamiento angular entre las palas de una turbina con respecto a la otra cambia de $0^{\circ}$ a $45^{\circ}$, entonces, con una velocidad de flujo de aire aproximadamente igual a $4.5 \mathrm{~m} / \mathrm{s}$, la potencia registrada aumenta entre el 3 y el 5 por ciento. Por supuesto, con una velocidad de flujo de aire diferente, este ángulo puede ser diferente. Además, se puede esperar que con la misma velocidad del flujo de aire exista un ángulo en que la potencia será máxima. Sin embargo, a pesar de un aumento en la potencia del aerogenerador relacionado con la rotación axial de las turbinas, una con respecto a la otra, los autores consideran que en la etapa actual,la implementación práctica de esto no es conveniente debido a que complica significativamente el diseño y el costo del aerogenerador.

\subsection{Diseño de las turbinas}

En proyectos modernos de aerogeneradores, tales como Vestas, Gamesa, Enercon, entre otros, se da preferencia a las turbinas equipadas con tres palas. Una de las razones de esta decisión, según la opinión de los autores del presente, es la siguiente: para un óptimo aprovechamiento de la energía cinética del viento mediante cambio del ángulo de ataque del flujo de aire, las turbinas están equipadas con un mecanismo de rotación de las palas con respecto a su eje longitudinal. El diseño de este mecanismo es más fácil de realizar para las turbinas equipadas con tres palas que para las turbinas con cuatro o más palas. En el diseño del aerogenerador presentado, el cambio del ángulo de ataque del flujo de aire en las palas no se necesita, ya que el control de la presión del flujo de aire en las palas de las turbinas, se realiza mediante el giro de la góndola en torno al eje $a$ $a$ de la torre, por consiguiente, las palas pueden fijarse en los cubos de las turbinas, por lo que el número de palas es menos limitado y depende del objetivo del diseñador. Por supuesto, la potencia del generador depende del número de palas, pero esta dependencia no es directamente proporcional. Si se toma un generador equipado con una turbina con cuatro palas y se compara con otro equipado con la turbina con dos palas, se puede suponer que el primero desarrollará el doble de potencia con respecto al segundo. Sin embargo, las pruebas del modelo en el túnel de viento mostraron que la potencia aumenta no más del 70 u 80 por ciento.

Era muy importante conocer el tamaño y la configuración de las palas. Se conoce la dependencia de la potencia del aerogenerador del diámetro de las turbinas, la cual se basa en el modelo de Betz presentado en la Fig. 4a. Se considera que la potencia de una turbina depende solamente del área de barrido de las palas, esta dependencia se ilustra en la Fig. 4b.

Es por eso que los fabricantes de aerogeneradores elaboran palas muy largas, por ejemplo, la empresa alemana Enercon para la turbina del aerogenerador Enercon-126 hace palas de longitud hasta 60 metros. La forma de las palas, condicionalmente, puede considerarse como trapezoidal. Esta forma se realiza teniendo en cuenta la resistencia a la flexión de las palas, la parte delantera de estas, se hace cóncava en su sección transversal, se supone que esto puede aumentar la eficiencia del uso de la energía cinética del viento.

Los autores asumieron que tanto la longitud de las palas (el diámetro de las turbinas) como su anchura afectan la eficiencia del uso de la energía cinética del viento. Para probar esta idea, se fabricaron varios juegos de las palas de diferente longitud y anchura, pero con la misma área. En estos experimentos, las palas se hicieron de forma rectangular y las mismas se torcieron de manera que el paso sea igual en todas las secciones transversales a lo largo de su longitud. De este modo se obtuvieron palas con forma de helicoide axial recto. En la Fig. 5 se muestran los resultados de las pruebas. Dichos resultados mostraron que las palas con una relación de la longitud $L$ a la anchura $B$ igual a $\lambda=L / B=8$ desarrollan una mayor potencia, por consiguiente tienen mayor eficiencia en el uso de la energía cinética del viento. 


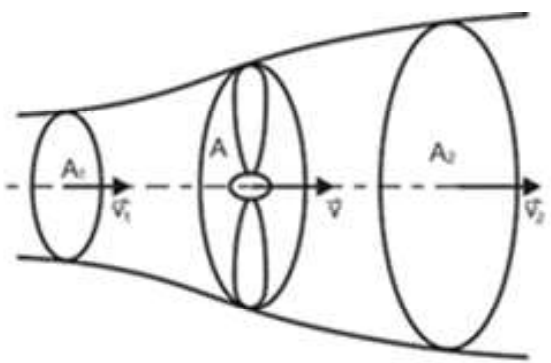

a)

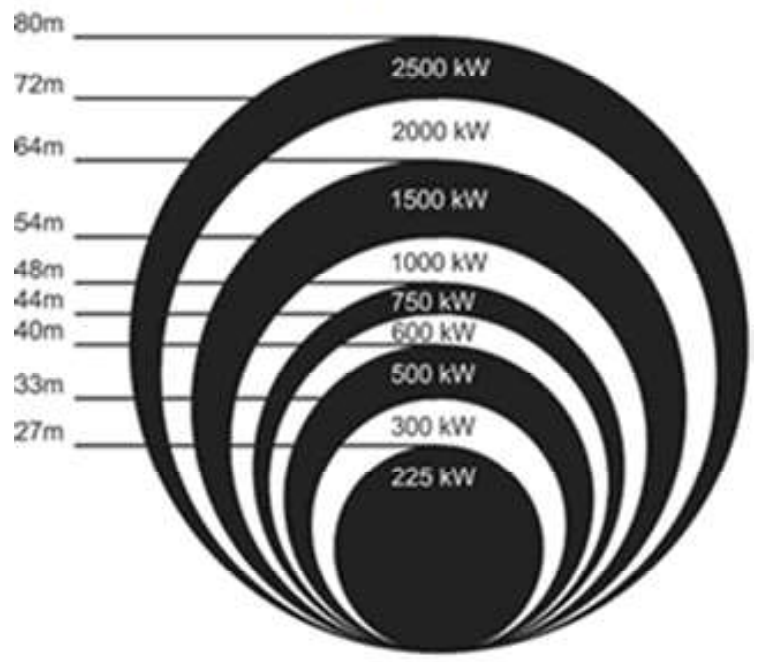

b)

Fig. 4. Modelo de Betz (a) y relación de la potencia de las turbinas de viento con su diámetro (b).

Curva de la dependencia de la eficienhcia del generador eólico equipado con dos turbinas del fact or $\lambda=\mathrm{L} / \mathrm{B}$

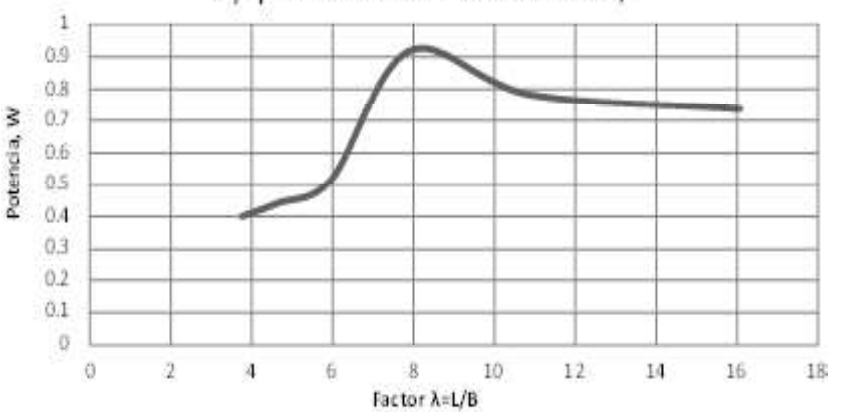

Fig. 5. Presentación gráfica de la dependencia de la potencia de la turbina del factor $\lambda$.
Además, los resultados mostraron que debido a la turbulencia creada por las partes cóncavas de las palas de la turbina de barlovento, la potencia de un generador equipado con dos turbinas era menor en comparación con la potencia esperada. Esta turbulencia llevó al hecho de que la velocidad de rotación de las turbinas no era constante y que tenía un cambio mayor que el observado en turbinas con palas planas. Probablemente debido a la misma turbulencia, no hubo aumento en la potencia de los generadores equipados con una sola turbina de barlovento equipada con palas cóncavas en su sección transversal.

Con base en las pruebas realizadas, se desarrolló el diseño de la pala [7], cuyo esquema se muestra en la Fig. 6. Las palas se hacen en dos partes: una parte delantera $10 \mathrm{y}$ la otra trasera 11. Dentro de las palas se colocan las costillas 12. Las costillas tienen forma cruzada y se unen a la parte delantera 10. La parte delantera 10 de las palas se hace recta en la dirección longitudinal que coincide con el eje $G-G$, o para compensar la deformación elástica de las palas causada por la fuerza del flujo de aire, ligeramente cóncava, como se muestra en la figura $6 \mathrm{~b}$. Las palas se hacen helicoidales, para esto se tuercen de modo que la línea de intersección de la superficie frontal de la parte 10 con el plano perpendicular al eje $G$ - $G$ de la pala, tenga el mismo paso en todas las secciones. De este modo se obtiene una pala con la superficie frontal de la parte 10 con forma de helicoide axial recto.

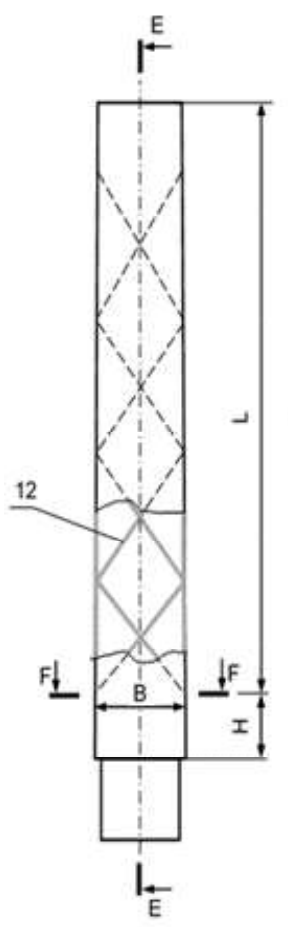

a)

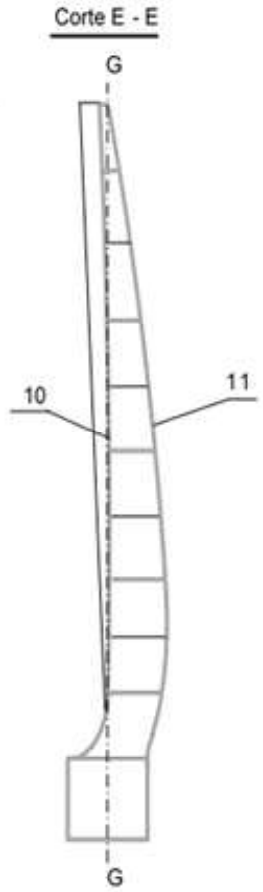

b)
Fig. 6. Presentación esquemática de la estructura de las palas. 
En la Fig. 7 se muestra un fragmento ampliado de la Fig. 2, donde las palas 9 están sujetas al cubo 13 de las turbinas de barlovento 4 y de sotavento 5 . La fijación de las palas 9 al cubo 13 se realiza de modo que la superficie frontal de la parte 10 , en la sección transversal $F-F$ (véase Fig. 6a), forme un ángulo $\beta$ con respecto al eje de rotación de las turbinas. Este ángulo, como regla, se toma igual a $45^{\circ}$.

La distancia $\mathrm{H}$ de la pala, que determina la posición de la sección $F-F$ desde la superficie del cubo 13, se elige constructivamente y depende del diseño de las turbinas 4 y 5. Para reducir la resistencia frontal al flujo de aire, la parte delantera de los cubos 13 está cubierta por los carenados 14 .

\subsection{Sistema de control automático de orientación de la góndola}

Bajo la acción del flujo de aire, las turbinas de los aerogeneradores pueden desarrollar una velocidad de rotación muy elevada, lo que provoca grandes fuerzas centrífugas capaces de destruir las palas de la turbina. Para reducir la velocidad de rotación de las turbinas en los aerogeneradores modernos, habitualmente se usa un freno de rozamiento, este emite una gran cantidad de calor, por lo que no asegura una alta confiabilidad y puede ser una fuente de ignición.

Para el modelo del aerogenerador que se presenta, no se requiere el uso de frenos, ya que la reducción de la velocidad de rotación de las turbinas se logra mediante el cambio de la posición angular de la góndola con respecto a la dirección del viento. El sistema de la variación automática de la posición de la góndola con respecto a la dirección del viento consta de una o dos pantallas 6 unidas a la varilla 7, la cual pasa a través de la góndola 3 en el lado de sotavento $M$, lo más cerca posible a la turbina de sotavento 5 (véase Fig. 1 y

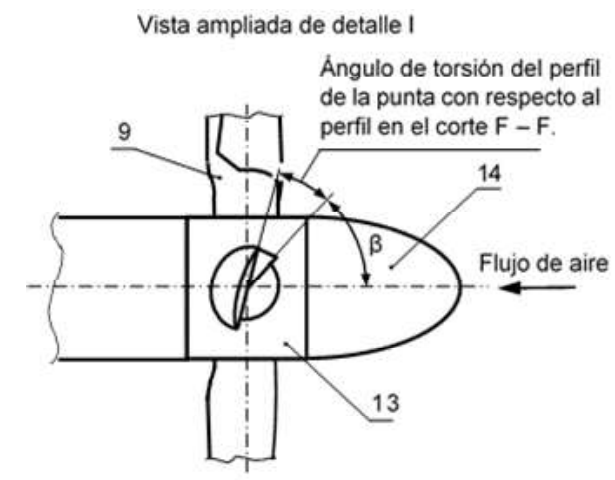

Fig. 7. Esquema de la fijación de las palas al cubo de la turbina.
Fig. 3). La varilla 7 cinemáticamente se conecta a un mecanismo de giro ubicado dentro de la góndola 3 (no se muestra en las figuras). Como mecanismo de rotación de las pantallas, se puede usar cualquier mecanismo de palanca o de engranes capaces de rotar la varilla 7 alrededor de su eje y, por lo tanto, cambiar la dirección del plano de las pantallas 6 . Una de estas soluciones se presenta en [5].

El sistema que cambia automáticamente la orientación de la góndola con respecto a la dirección del viento funciona de la siguiente manera: con una velocidad de viento aceptable, el mecanismo orienta las pantallas 6 paralelamente al eje longitudinal del cuerpo de la góndola 3. Como las pantallas 6 están instaladas en el lado de sotavento de la góndola, entonces estas van a actuar como un timón de cola rígido que orienta la góndola 3 paralelamente al flujo de aire. Los momentos de fuerzas provenientes de las turbinas, al actuar en la góndola se equilibran entre sí, ya que están ubicadas en los extremos opuestos de la góndola 3 y tienen la misma magnitud y sentido, por lo que no afectan la orientación de la góndola. Si la velocidad del viento excede lo permitido para el seguro funcionamiento del aerogenerador, el sensor de velocidad dará un comando para cambiar la orientación de la góndola 3. En este caso, el mecanismo de rotación de la varilla 7 rotará las pantallas 6, creando así una resistencia adicional al flujo de aire. Dado que las pantallas 6 están instaladas en el lado de sotavento de la góndola 3, el viento que actúa sobre las pantallas 6 , generará un momento de fuerza aplicado a la góndola 3, girándola en un ángulo con respecto a la dirección del viento. Por lo anterior, el flujo de aire actuará en las turbinas en un ángulo menor a $90^{\circ}$, lo que disminuirá la velocidad de rotación de las turbinas 4 y 5 . En casos extremos, cuando la velocidad del viento sea tal que las fuerzas centrífugas que actúen sobre las palas 9 puedan destruirlas, las pantallas 6 se instalarán perpendicularmente a la góndola 3 , entonces los planos de rotación de las turbinas 4 y 5 se instalarán paralelamente al flujo del aire, y la rotación de las turbinas 4 y 5 se detendrá. Dado que la resistencia aerodinámica de las partes de barlovento $\mathrm{K}$ y de sotavento $\mathrm{M}$ de la góndola 3 son iguales, entonces lo único que determinará la posición de la góndola serán las pantallas 6 . Cuando se registre una disminución en la velocidad del viento, la señal del sensor de velocidad dará comando para cambiar la posición de las pantallas 6 , y el mecanismo de rotación de las pantallas 6 las regresará en la posición de inicio, por lo tanto, la góndola 3 retomará una posición paralela al flujo de aire.

\subsection{Multiplicador de la velocidad de rotación de la flecha del generador eléctrico}

Las turbinas de los aerogeneradores, en general, tienen una velocidad de rotación pequeña, aproximadamente de 5 a 10 min-1 pero la velocidad de rotación de la flecha de un genera- 
dor eléctrico estándar convencional debe ser de al menos 300 min-1. Por lo tanto, para generar la energía eléctrica deseada, los aerogeneradores están equipados con multiplicadores de velocidad, que esencialmente se pueden hacer en forma de reductores cilíndricos convencionales, en los que la flecha motriz es la flecha de baja velocidad y la flecha impulsada es de alta velocidad. En la Fig. 8 se muestra el esquema de un multiplicador de velocidad de alto rendimiento [6]. Este esquema es válido para el caso cuando las dos flechas del aerogenerador son motrices. Dentro de la góndola 3, en el soporte 15 está montada la flecha 16 sobre la cual está instalada la turbina de barlovento 4, mientras que en el soporte 17 está montada la flecha 18 sobre la cual está instalada la turbina de sotavento 5. Para que ambas flechas tengan la misma velocidad y sentido de rotación, estas están unidas por un acoplamiento 19. Dentro de la góndola, en las puntas de las flechas 16 y 18 , se montan los engranes cónicos 20 y 21 , respectivamente. Con el engrane 20 se engrana la rueda dentada 22 , y con el engrane 21 la 23.

Los engranajes están diseñados de modo que el engranaje de las ruedas 21 con 23 tenga dimensiones menores que el de la 20 con la 22 , y el eje geométrico de la rueda 23 coincida con el eje de la rueda 22 . Además, la flecha en la rueda 22 es hueca, de modo que la flecha de la rueda 23 puede instalarse en esta cavidad. La flecha de la rueda 23 se conecta con el brazo 24 del multiplicador, y la flecha de la rueda 22 con la carcasa 25 del multiplicador. El satélite 26 está montado en el brazo 24 y a la vez está engranado con el engrane sol 28 y con la corona 27. La corona 27 se fija con la carcasa 25 , formado de tal

\section{Vista del corte D-D}

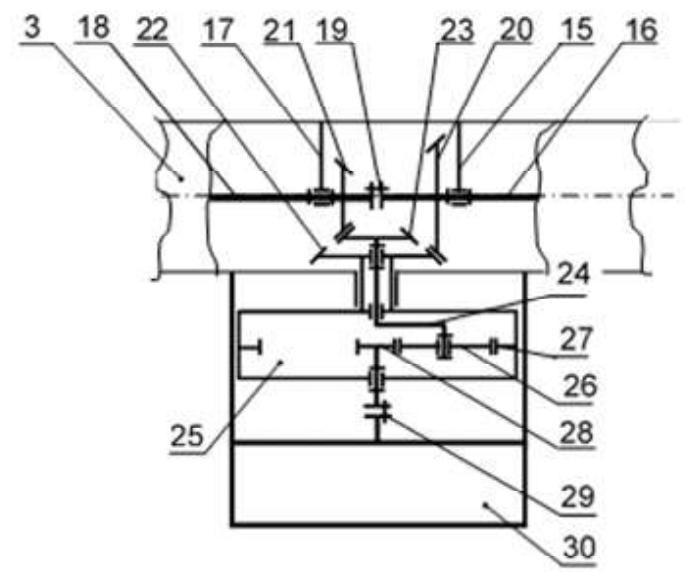

Fig. 8. Esquema del multiplicador de velocidad. manera un solo eslabón. La flecha del engrane sol 28 mediante el acoplamiento 29 se conecta con la flecha del rotor del generador eléctrico 30 .

Bajo la acción del flujo de aire, las turbinas 4 y 5 giran en el mismo sentido y con la misma velocidad angular, transmitiendo el movimiento giratorio a las flechas 16 y 18 , respectivamente. Por lo tanto, los engranajes cónicos 20 y 21 también girarán en el mismo sentido y con la misma velocidad angular. Dado que los acoplamientos de las ruedas 20 con 22 y 21 con 23 con respecto a los ejes geométricos de las ruedas 22 y 23 están en el sentido opuesto, las ruedas 22 y 23 girarán en sentidos opuestos. Por lo tanto, el brazo 24 y la carcasa 25 del mecanismo van a girar en sentidos opuestos. El mecanismo multiplicador que se muestra en la Fig. 8 es un mecanismo diferencial cerrado. Un mecanismo diferencial cerrado es un mecanismo diferencial con dos grados de libertad (en el que todos los eslabones son móviles), en el que dos eslabones móviles están cerrados (conectados entre sí) por una cadena cinemática ordinaria con los ejes inmóviles de los engranajes (en la Fig. 8 es un engranaje de la rueda 20 con 22 y 21 con 23). La cadena cinemática ordinaria con los eslabones con ejes inmóviles transforma un mecanismo diferencial con dos grados de libertad en un mecanismo diferencial cerrado con un solo grado de libertad y, por lo tanto, con un solo eslabón de entrada, que es el brazo 24, y un eslabón de salida, que es el engrane sol 28.

En la Fig. 9 se muestra la justificación gráfica de funcionamiento del mecanismo. En la Fig. 9a se muestra el esquema cinemático del mecanismo y en la Fig. $9 \mathrm{~b}$ el polígono vectorial de velocidades de los puntos característicos de los eslabones del mecanismo.

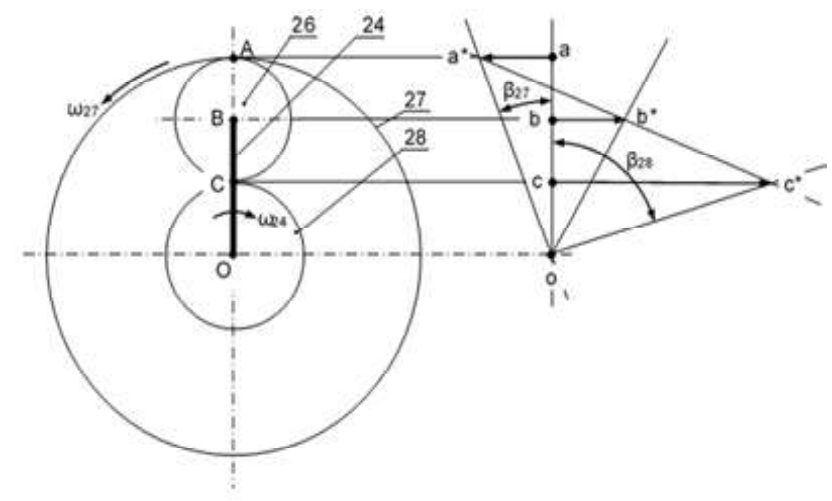

Fig. 9. Presentación del funcionamiento del multiplicador de velocidad mediante el análisis del polígono vectorial de velocidades. 
Al suponer que la relación de transmisión de los pares de engranes cónicos del 20 con 22 y del 21 con 23 es igual a uno:

$$
e_{20,22}=\frac{z_{22}}{z_{20}}=1 \quad \text { y } e_{21,23}=\frac{z_{23}}{z_{21}}=1
$$

Entonces la corona 27 tomará una velocidad angular igual a la velocidad angular de la rueda $22 \mathrm{y}$, por lo tanto, igual a la velocidad angular de la turbina de barlovento 4 , mientras que el brazo 24 tomará una velocidad angular igual a la velocidad angular de la rueda $23 \mathrm{y}$, por lo tanto, igual a la velocidad angular de la turbina de sotavento 5. Ya que el satélite 26 está engranado con la corona 27 , entonces el punto A del satélite 26 tendrá una velocidad tangencial igual a la velocidad del punto A de la corona 27, y el punto B del mismo satélite tendrá una velocidad tangencial igual al punto B del brazo 24 .

Suponiendo que la velocidad del punto A del satélite 26 es igual al vector $\overline{\mathrm{a}-\mathrm{a}^{*}}$ y el punto $\mathrm{B}$ del mismo satélite 26 es igual al vector $\overline{b-b^{*}}$ entonces la línea $\overline{a^{*}-b^{*}}$ será la línea de distribución de las velocidades de los puntos del satélite 26 , lo que significa que el punto $\mathrm{C}$ del satélite 26 tendrá una velocidad igual al vector $\overline{\mathrm{c}-\mathrm{c}^{*}}$. Dado que el satélite 26 está unido simultáneamente con la rueda sol 28 , entonces el punto $\mathrm{C}$ de la rueda sol 28 tendrá una velocidad igual al vector $\overline{\mathrm{c}-\mathrm{c}^{*}}$.

La velocidad angular de un eslabón se puede representar en forma $\omega=v / r$, donde $v$ es la velocidad tangencial de un punto del eslabón, y $r$ es la distancia del punto de aplicación de la velocidad $v$ desde el centro de rotación del eslabón. Por lo tanto, en forma vectorial, la velocidad angular de la rueda 27

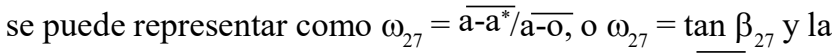
velocidad angular de la rueda sol 28 como $\omega_{28}=\overline{\mathrm{c}-\mathrm{c}^{*} / \mathrm{c}-\mathrm{o}, \mathrm{o}}$ $\omega_{28}=\tan \beta_{28}$.

En la figura $9 \mathrm{~b}$ se puede observar que el ángulo $\beta_{28}$ es significativamente mayor que el ángulo $\beta_{27}$, por lo que la velocidad de rotación de la flecha del generador eléctrico será mucho mayor que la velocidad de rotación de las turbinas 4 y 5 .

\section{Conclusiones}

Resumiendo el material presentado, se puede concluir que se ha creado un nuevo modelo del aerogenerador que, en comparación con los que se utilizan actualmente en la industria energética, tiene las siguientes ventajas:

1. Con la instalación de dos turbinas, en los extremos de la misma góndola una de barlovento y otra de sotavento, la potencia del generador de viento se puede aumentar al menos en un 40 por ciento en comparación con el uso de una turbina de barlovento.
2. El uso de las palas en forma de helicoide axial recto con una razón de la longitud a la anchura aproximadamente igual a 8 , reduce significativamente la longitud de las palas, lo que reduce su costo tanto en la fabricación como en el transporte.

3. El costo del aerogenerador presentado también se puede reducir al simplificar el diseño, ya que el generador presentado no utilizará freno de rozamiento para reducir la velocidad de rotación de las turbinas. Tampoco utilizará el mecanismo de rotación de las palas alrededor de su eje longitudinal, ya que la velocidad de rotación de las turbinas se varia mediante la variación automática de la orientación de la góndola con respecto a la dirección del viento. Por esto mismo, el aerogenerador presentado es más confiable y seguro en su funcionamiento. Además, el modelo presentado de aerogenerador puede producir energía eléctrica en un rango muy amplio de velocidades del viento, desde $3 \mathrm{~m} / \mathrm{s}$, hasta $25-30 \mathrm{~m} / \mathrm{s}$. Esto se debe al hecho de que en cualquier posición de la góndola con respecto a la dirección del viento puede seguir operando y generando energía eléctrica en su potencia nominal.

Se puede argumentar que la producción de dos turbinas para un aerogenerador es costosa. Sin embargo, los autores creen que la producción de la turbina extra se compensa significativamente con la simplificación del diseño del aerogenerador y con la mayor confiabilidad en su funcionamiento. Además, el nuevo aerogenerador requiere poca energía eléctrica para ejecutar la rotación de la góndola, ya que el viento mismo hace girar la góndola.

\section{Referencias}

[1] B. F. Voronin, Generador eólico de eje horizontal con dos turbinas instaladas en extremos de la góndola con el control automático de la posición de la góndola con respecto a la dirección del viento. Solicitud de registro de modelo de utilidad No. MX/u/2019/000109, 2019.

[2] B. F. Voronin, J. A. Gómez-Reyna, M. D. ZerqueraIzquierdo et al., Conceptos principales del uso de energía eólica en México. XVI Congreso Nacional de Ahorro de Energía, Tlaquepaque, México, 2005.

[3] B. F. Voronin, J. A. Gómez-Reyna, M. D. ZerqueraIzquierdo et al., Un nuevo generador eólico para México. Energía Racional, núm. 73, oct-dic 2009, pp. 3-9.

[4] B. F. Voronin, J. A. Gómez-Reyna, M. D. ZerqueraIzquierdo et al., Investigación de generadores eólicos mediante un modelo a escala. XXI Congreso Internacional de Ahorro de Energía, Guadalajara, 2010. 
[5] B. F. Voronin, Mecanismo de orientación de un generador eólico con dos turbinas.Solicitud de patente RU2014138384 (A) -2016-04-10,2016.

[6] B. F. Voronin, Generador eólico con dos turbinas de palas planas. Invención internacional registrada, núm. WO2010/
087689 (PCT/MX2009/000008), 2009.

[7] B. F. Voronin, V. R. Nuño-Sánchez, Generador eólico con dos turbinas de palas con forma de helicoide axial recto, instaladas en los extremos de la góndola. Solicitud de patente MX/u/2017/000588, 2017. 
